\title{
CT Image Reconstruction Algorithm to Reduce Metal Artifact*
}

\author{
Michihiko KOSEKI**, Shuhei HASHIMOTO ${ }^{* * *}$, Shimpei SATO ${ }^{\dagger}$, \\ Hitoshi KIMURA** and Norio INOU** \\ ** Graduate School of Science and Engineering, Tokyo Institute of Technology \\ 2-12-1, O-okayama, Meguro-ku, Tokyo, 152-8550 Japan \\ *** Nissan Motor Co., Ltd. \\ 560-2, Okatsukoku, Atsugi-shi, Kanagawa, 243-0192, Japan \\ $\dagger$ Central Japan Railway Company \\ 2-1-85, Konan, Minato-ku, Tokyo, 108-8204, Japan
}

\begin{abstract}
Micro computed tomography $(\mu \mathrm{CT})$ is quite useful in the nondestructive evaluation of devices with complicated internal structures. However, a large difference in X-ray absorption coefficients of the materials produces streak and star pattern artifacts in a CT image. These artifacts, which are called "metal artifacts", make it difficult to inspect an image of the device. Hence, this study strives to develop a new reconstruction method that reduces the artifacts from images. In this paper, we first discuss the cause of the artifacts using CT data of a sample. Next, we propose a CT reconstruction algorithm to reduce the artifacts. The basic idea of the algorithm is that it corrects the projection data. The shapes of high density parts such as metals are extracted by a threshold method and projection data are replaced to consistent values. Finally, we apply the method to select devices, and confirm that the method is applicable to nondestructive inspections.
\end{abstract}

Key words : Nondestructive Inspection, Shape Measurement, Image Processing, X-Ray Computed Tomography, Metal Artifact, Inverse Problem

\section{Introduction}

Currently, electro-mechanical systems and devices are composed of various materials. Hence, a non-destructive inspection technique is necessary in production fields because the commercial values of these devices depend on not only the machining accuracy of each part, but also the assembly accuracy of the entire system. For example, consider a lens unit for a digital camera. The unit is composed of multiple materials, such as resins, glasses, metals, ceramics, and so on. It seems that the primal inspection of the lens unit is an evaluation of the condition of the resin parts. Thus, during the manufacturing processes, an inspection to detect voids in the resin parts is important. In addition, for quality certification of the unit, other items must be inspected for issues such as thermal or structural damage after testing for fatigue.

To evaluate the quality of a device, micro x-ray computed tomography $(\mu \mathrm{CT})$ has the potential to become a great efficient technique because it provides three-dimensional geometrical information of the internal structures. However, hurdles remain in applying the CT imaging technique to inspect devices. That is, a large difference in x-ray absorption coefficients of materials sometimes produces artifacts with streak and star patterns in the CT images, especially when CT scanning is performed with low intensity x-rays. In fact, low intensity x-rays must be employed to inspect the lens unit due to the smaller x-ray absorption coefficient of the resin. In this case, artifacts in CT images are inevitable. The artifacts, which are generally called "metal artifacts," disturb the detailed observation of the resin part. 
This study aims to establish a highly accurate non-destructive inspection technique for devices with various materials using x-ray CT imaging. Our initial study examined the accuracy of medical CT images, and proposed correction methods for the partial volume effect, which is caused by the thickness of the $\mathrm{x}$-ray beam ${ }^{(1)}$,(2). This effect provides ambiguous CT images, especially at the surface of a scanning subject. Although previous methods successfully correct the partial volume effect, these methods are ineffective for CT images with metal artifacts. In the next stage of our study, we focused on artifacts in medical CT images because numerous people now have metallic implants in their bodies. We attempted to reduce the artifacts caused by dental fillings or implants in order to obtain the exact material properties of bones and soft tissues from CT images ${ }^{(3),(4)}$. The phenomenon of metal artifacts in medical CT images is essentially no different from that of artificial devices.

This paper proposes a new CT reconstruction algorithm that provides accurate CT images without metal artifacts, regardless of the materials of the device. The details of the algorithm are explained using a sample with a simple structure, while the efficiency of the algorithm is discussed based on an application to an actual electrical device.

\section{Overview of $\mathrm{x}$-ray $\mathrm{CT}$ imaging and previous studies}

Cross-sectional CT images are generally reconstructed from $\mathrm{x}$-ray projections of the object ${ }^{(5)}$. Projection data is generated using an $\mathrm{x}$-ray source that rotates around the object with $\mathrm{x}$-ray sensors positioned on the opposite side of the circle from the $\mathrm{x}$-ray source. Pixels in a medical CT image are displayed in terms of relative radio-density. The brightness of the pixel, called the CT value, is proportional to the mean attenuation of the material, which corresponds to the Hounsfield scale. Water has an attenuation of 0 Hounsfield units (HU), whereas air has a value of $-1000 \mathrm{HU}$. The computation of a Hounsfield unit is performed using a calibrator filled with water. However, it is not necessary to perform such a calibration in the case of industrial x-ray CT scanning, and thus, we treat the pixel values of industrial CT images as indicators of the materials.

An x-ray CT image has metal artifacts when the object contains materials with extremely high x-ray attenuation coefficients like metals, which frequently occur during industrial inspections. Several reconstruction techniques have been proposed to reduce metal artifacts from medical CT images ${ }^{(6)-(10)}$. These techniques can be roughly classified into three methods, and their applications to nondestructively inspect artificial devices are discussed below.

The first method reconstructs a cross-sectional image based on an iterative calculation such as an expectation maximization (EM) method or an algebraic reconstruction technique $(\mathrm{ART})^{(6),(7)}$. The method requires massive calculation power, and is difficult to apply to general CT devices due to the mandatory calculation time for imaging.

The second method is used for visualization to guide cryosurgery ${ }^{(8)}$. Cryosurgery is a therapeutic procedure to ablate tissue by freezing an injury. The proposed method in this report uses two CT images: one scanned before the cryoprobes are inserted, and the other after the cryoprobes are put into place. Hence, it is difficult to apply this method to non-destructive inspections.

The third method corrects $\mathrm{x}$-ray projection data when $\mathrm{x}$-rays transmit high-density materials ${ }^{(9),(10)}$. The method localizes the metallic objects in the original CT image using a simple thresholding of the CT numbers in the first procedure. Then, the extracted image of the metallic objects is provided for the forward projection in order to obtain an ideal absorption by the object. Next, the actual projection data is refined using the forward projected data. Although this method significantly improves image quality, the interpretation of the first step is debatable. That is, the original CT image with metal artifacts is insufficient to obtain accurate shapes and orientations of metallic objects.

Our method proposed herein improves upon the third method in order to resolve the aforementioned problem. The unique feature of our method is based on a direct signal conversion technique for the projection data. A detailed description of the method is described 


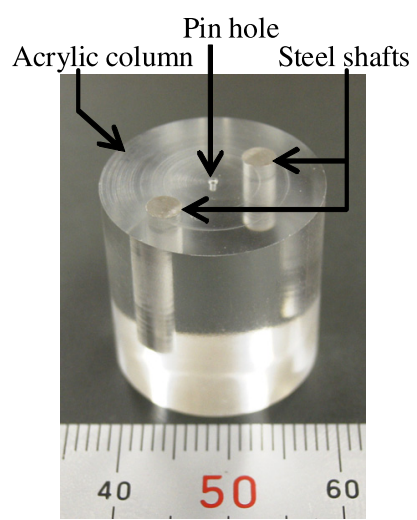

(a) Sample for CT imaging.

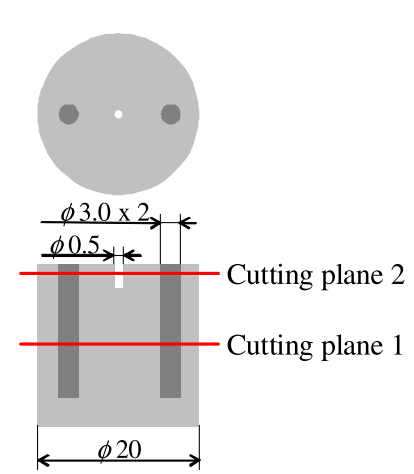

(b) Schematic diagram of the sample.

Fig. 1 Sample for CT imaging.

below using the sample shown in Fig.1(a).

The sample was composed of an acrylic column with a $20 \mathrm{~mm}$ diameter and two steel shafts with $3 \mathrm{~mm}$ diameters. At the center of the column, the sample had a hole $0.5 \mathrm{~mm}$ in diameter and $5 \mathrm{~mm}$ in depth. CT scannings of the sample were performed by a micro-focus x-ray CT scanner (ScanXmate-A130SS940, Comscantecno Co., Ltd.). The scanner had a matrixtype $\mathrm{x}$-ray sensor, which was comprised of $640 \times 480$ detectors. Hence, the scanner was able to perform cone-beam $\mathrm{x}$-ray radiations while simultaneously providing three-dimensional projection data. In this study, we treated the scanner like a fan-beam CT scanner using the outputs of the central $512 \times 1$ detectors due to the difficulties of three-dimensional reconstruction. The sample was provided to the $\mathrm{CT}$ scanning under the following conditions: the tube voltage on the $\mathrm{x}$-ray source $=80 \mathrm{kV}$, and the tube current $=3 \mu \mathrm{A}$. X-ray radiations to the sample were performed from 800 directions in a 360-degree rotation (rotation resolution is 0.45 ). CT scannings were performed at two cutting planes as illustrated in Fig.1(b); one was at the center of the column (Cutting plane 1), and the other was in the upper region with the small hole (Cutting plane 2).

Figure 2(a) shows the projection data at the cutting plane 1. The figure shows the data in a 180-degree rotation due to its symmetrical property. We initially reconstructed the crosssectional image of the sample using a conventional $\mathrm{CT}$ reconstruction algorithm, Filtered Back Projection (FBP). As shown in Fig.2(b), the reconstructed image has heavy metal artifacts, which make it extremely difficult to inspect the states of the resin parts.

\section{CT image reconstruction algorithm to reduce metal artifacts}

To thoroughly examine the cause of the metal artifacts, we plotted the profiles of the x-ray attenuation at rotation angles $r=90^{\circ}$ and $r=180^{\circ}$ for the projection data shown in Fig.2(a). Figure 3 shows the profiles. In these graphs, the horizontal axes indicate the detector number $d$, while the vertical axes represent the output values of each detector corresponding to x-ray absorption. The output values were about 0.5 in regions where $\mathrm{x}$-rays transmitted only the acrylic resin. On the other hand, the values in the steel shafts regions were five or more times higher. Furthermore, there were few differences in the output values between the regions that x-rays transmitted one shaft $(d \approx 150$ and $d \approx 360$ in Fig.3(a)) and two shafts $(d \approx 256$ in Fig.3(b)), despite a linear relationship for the output value and the number of the shafts is reasonable. This means that the projection data contains inconsistencies. Conventional CT reconstruction algorithms such as FBP are based on coherent x-ray absorption by the object. Thus, the main cause of the metal artifacts seems to be inconsistent projection data.

To reduce metal artifacts in $\mathrm{CT}$ images, our CT reconstruction algorithm corrects the inconsistencies in the projection data. That is, the algorithm extracts projection data corresponding to metallic objects and replaces the data to a consistent value. This method is 


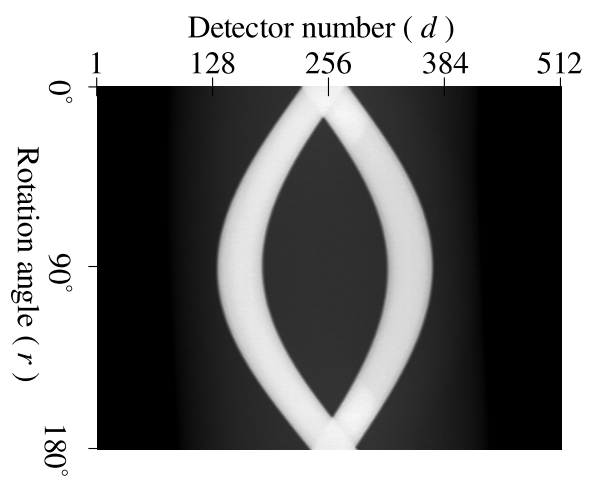

(a) Projection data of the sample at cutting plane 1 .

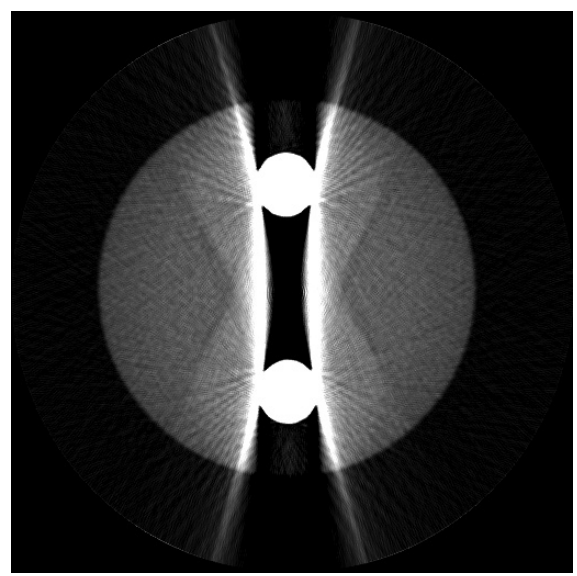

(b) Reconstructed image of the sample.

Fig. 2 Projection data and reconstructed image of the sample.

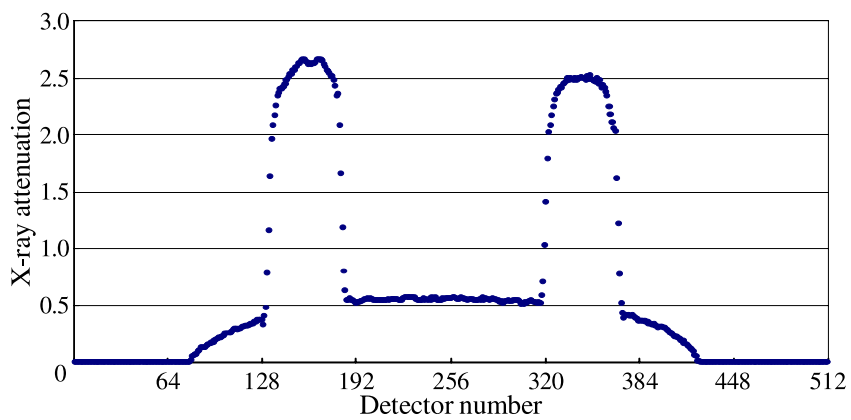

(a) $r=90^{\circ}$

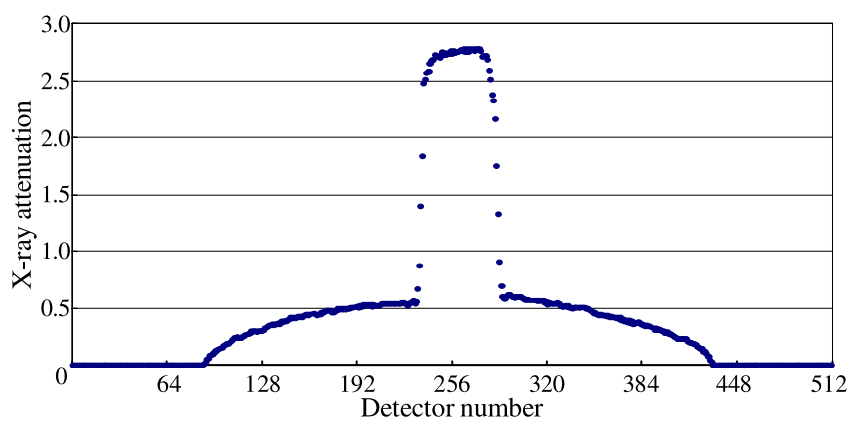

(b) $r=180^{\circ}$

Fig. 3 Profiles of the X-ray attenuation (original.) 


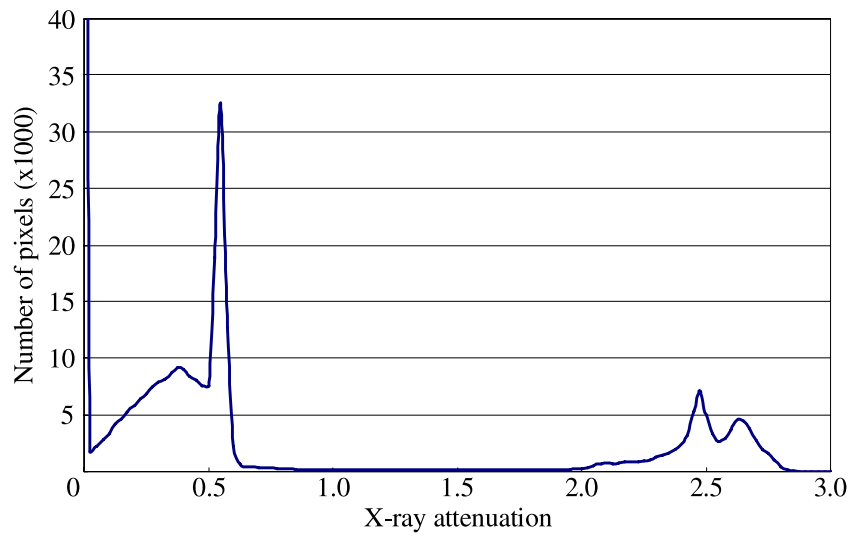

Fig. 4 Histogram of the X-ray attenuation.

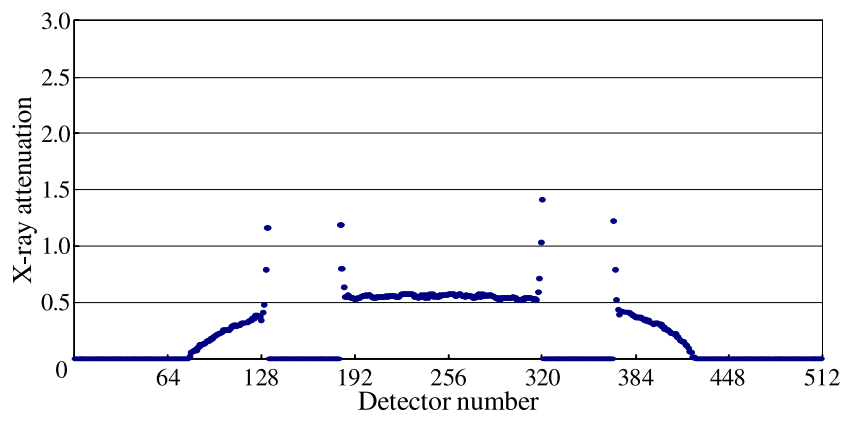

Fig. 5 Profile of the X-ray attenuation (Step 1.)

composed of the following five steps:

Step 1. Rough extraction of the metal regions from the projection data.

Step 2. Determination of the metal regions.

Step 3. Computation of the cross-sectional shapes of the metallic objects.

Step 4. Calculation of the numerical projection data of the metal shape.

Step 5. Synthesis of the measured and numerical projection data.

A detailed description of the method is described below using the profile of the projection data at $r=90^{\circ}$.

Step 1: Rough extraction of the metal regions: We initially extract the region where the x-ray transmits high-density materials from the projection data using a threshold. The threshold value is determined from a histogram of the x-ray absorption. Figure 4 shows the histogram of this example. The large frequencies at attenuation values lower than 0.8 indicate $\mathrm{x}$-ray transmissions of air or of an acrylic resin part. On the other hand, there are also large frequencies at an attenuation value of about 2.5 , which indicate $\mathrm{x}$-ray transmissions of metallic objects. That is, a threshold between 1.0 and 2.0 separates metallic objects from the rest. In this case, we set 1.5 as the threshold, and we modified the x-ray attenuation to 0.0 where the value is larger than the threshold. Figure 5 shows the computed result for this step. Although we manually decided the threshold value in this study, we can use the automatic threshold selection method reported by Otsu ${ }^{(11)}$.

Step 2: Determination of the metal regions: The extracted data in the step 1 remains in transitional regions, which depend on the threshold value. Then, we discriminate these regions using derivative values of the projection data. We adopt the two following derivatives in consideration of measurement noises:

$$
\begin{aligned}
& D_{1}=|f(d+1, r)-f(d, r)| \\
& D_{2}=|f(d+1, r)-f(d-1, r)|
\end{aligned}
$$

Here, $f(d, r)$ is projection data at x-ray detector number $d$ and rotation angle $r$. We determine 


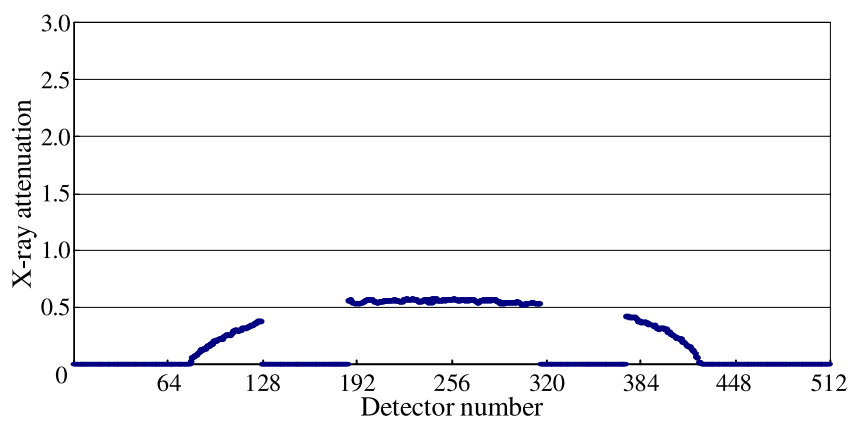

Fig. 6 Profile of the X-ray attenuation (Step 2.)

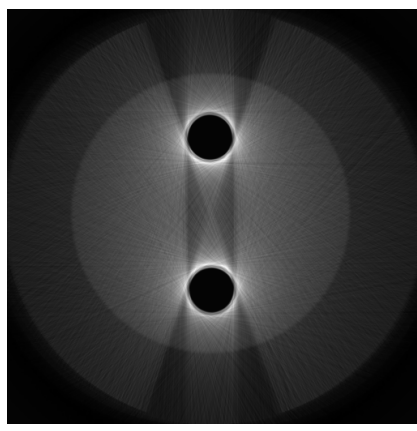

(a) CT image without steel shafts

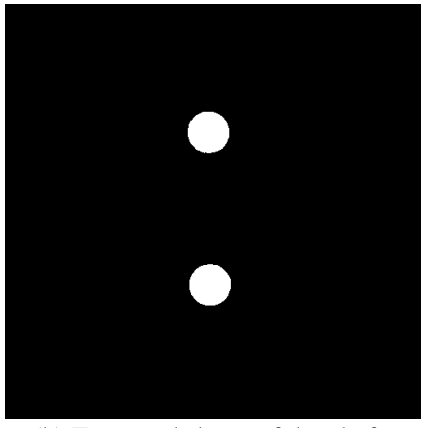

(b) Extracted shape of the shafts

Fig. 7 Extraction of the shape of the steel shafts.

metal regions with larger $D_{1}$ and $D_{2}$ than each average value. Figure 6 shows the result of this step.

Step 3: Computation of the cross-sectional shapes: The modified projection data in steps 1 and 2 is provided to the back projection and a cross-sectional image is computed. Because the metal regions in the projection data are set to zero in the previous steps, the pixels corresponding to metallic objects also indicate zero in the image. Thus, we can easily extract the shapes and locations of the metallic objects in the image. Figure 7(a) shows the obtained image in this step, and Fig.7(b) shows the extracted shapes of the metallic objects from the image.

Step 4: Calculation of the numerical projection data: A forward projection is applied to the computed image of the shapes of the metallic objects obtained in the step 3. This is equivalent to numerical x-ray radiation of the metallic objects. In the forward projection, we can configure the lower $\mathrm{x}$-ray attenuation coefficient $\mu$ to the metal region compared to the actual coefficient. In order to adjust the x-ray attenuation by the metal region to a value comparable in magnitude to it by the resin region, we set $\mu=0.01$ as the coefficient of the metallic object. Figure 8 shows the numerical projection data of the metal region shown in Fig.7(b). Because the configured coefficient $\mu$ of the metal region is one of the important parameters affecting the accuracy of the reconstructed images, it is preferable to select the appropriate value according to the complexities or materials of the objects. However, the proposed method seems to be robust for the coefficient $\mu$ because the cause of the metal artifacts is inconsistent in the projection data, but the proposed method computes consistent data regardless of $\mu$. Our future study will quantitatively evaluate the relationship between the coefficient $\mu$ and the accuracy of the image.

Step 5: Synthesis of the measured and numerical data: This step synthesizes the projection data modified in the steps 1,2 , and the computed projection data in the step 4 . Initially the metal regions in the modified projection data are linear interpolated as shown in Fig.9. Then the computed projection data (Fig.8) are added up to the data. Figure 10 shows rectified projection data. Because this projection data indicate an almost ideal attenuation, the data are anticipated to reconstruct a cross-sectional image without metal artifacts. 


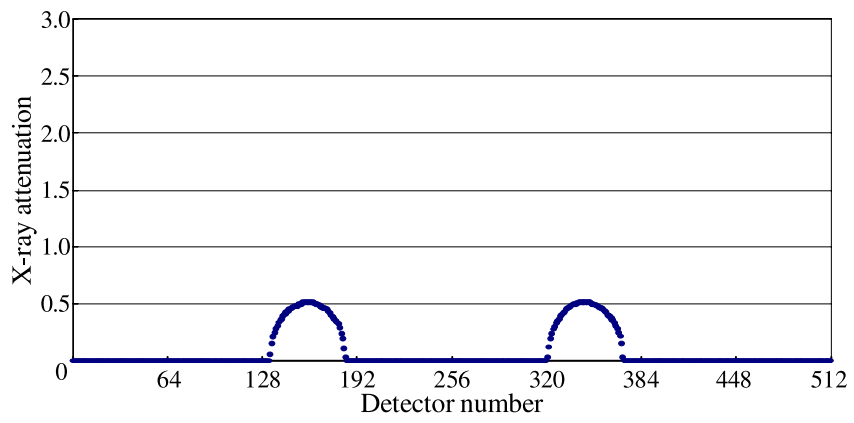

Fig. 8 Profile of the X-ray attenuation (Step 4.)

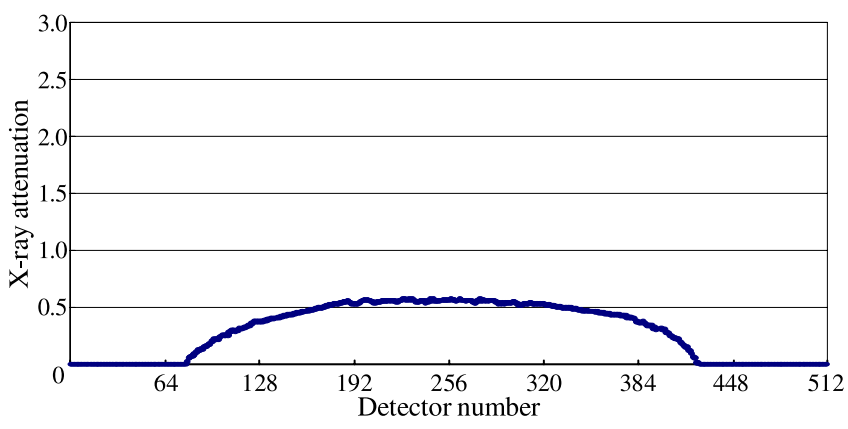

Fig. 9 Profile of the X-ray attenuation (Step 5.)

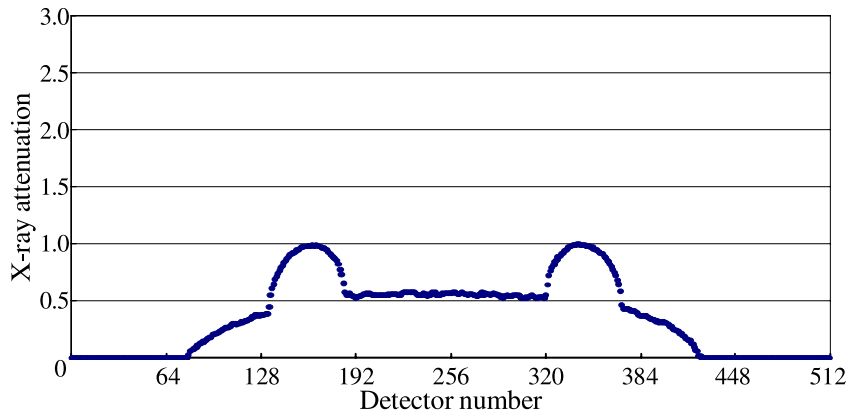

(a) $r=90^{\circ}$

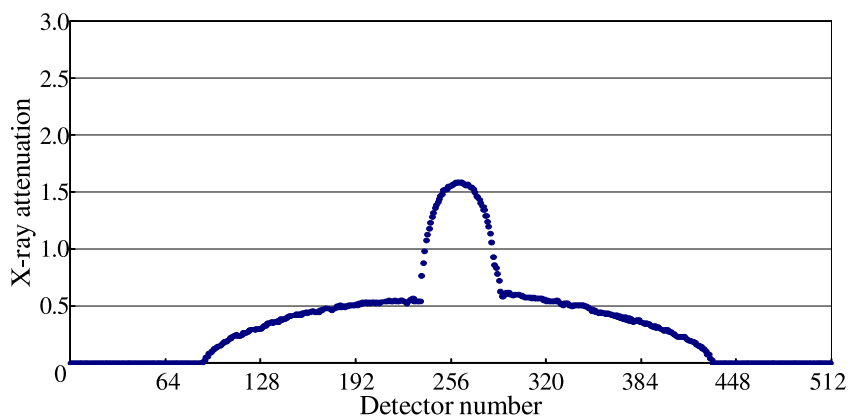

(b) $r=180^{\circ}$

Fig. 10 Profile of the X-ray attenuation (revised.) 


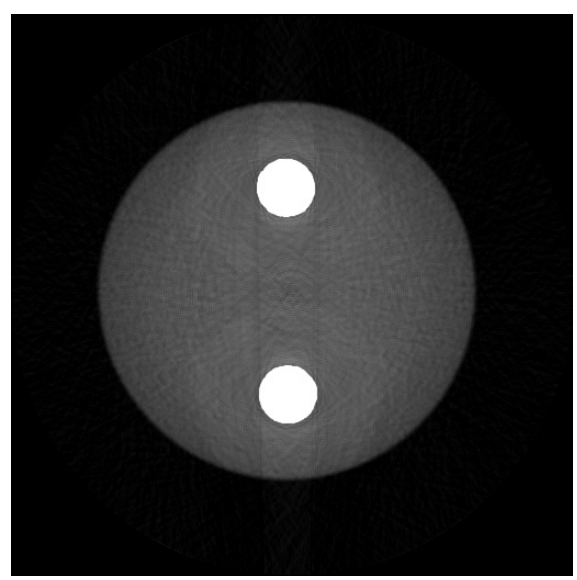

Fig. 11 Reconstructed images of the sample.

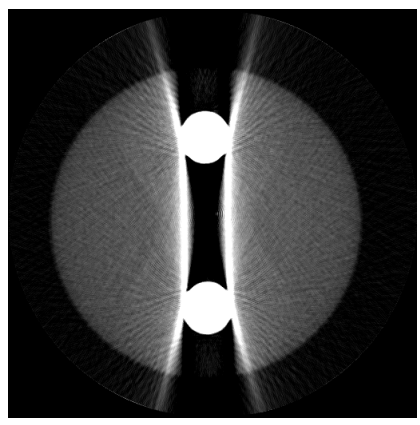

(a) Reconstructed image using the original projection data.

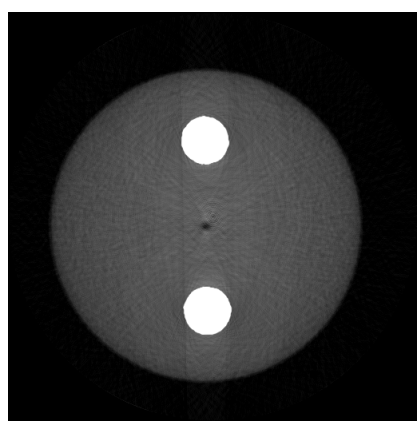

(b) Reconstructed image using the revised projection data.

Fig. 12 Reconstructed images of the sample.

\section{Reconstructed CT images and accuracy evaluation}

The proposed method was applied to cutting plane 1 of the sample shown in Fig.1. Figure 11 presents the reconstructed image of the revised projection data. In this research, we employed an industrial x-ray CT scanner, so the brightness of each pixel does not indicate the Hounsfield unit as mentioned above. Then, the brightness of Fig.11 was adjusted to be almost same as the original image (Fig.2(b)) in the resin region. The reconstructed image based on the original projection data had heavy metal artifacts as shown in Fig.2(b). On the contrary, the reconstructed image using the proposed method had few metal artifacts, as shown in Fig.11. Consequently, the states of the resin part are easily observed.

Next, we applied the method to cutting plane 2 of the sample. In this plane, the sample had a small hole at the center. Figure 12(a) shows the reconstructed image using the original projection data, while Fig.12(b) shows the image based on the revised data. The image using the original data had artifacts similar to Fig.2(b), the pixel values in the region between the two shafts were comparable to that of the air. It is impossible to inspect the region with this image. On the other hand, the proposed method reconstructed an accurate image, which enabled the hole between the shafts to be observed. This suggests that the proposed method is efficient as a non-destructive inspection technique such as void detection in the manufacturing process or damage analysis in fatigue testing.

Finally, to evaluate the efficiency for practical use, the method was applied to an electrical connector with three terminal pins, which is shown in Fig.13. In this case, x-rays for scanning were provided as the source with a tube voltage $=50 \mathrm{kV}$ and a tube current $=110 \mu \mathrm{A}$. The remaining scanning conditions were the same as those of the previous specimen. Figure 14 depicts the reconstructed images. The image based on the original projection data had metal artifacts, and it is hard to discriminate the shape of resin around the terminal pins as 


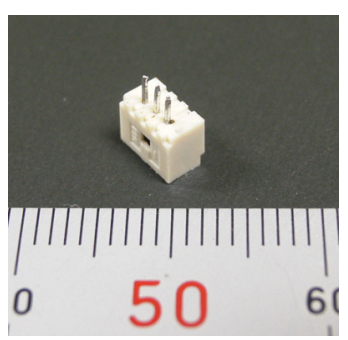

(a) A connector for CT imaging.

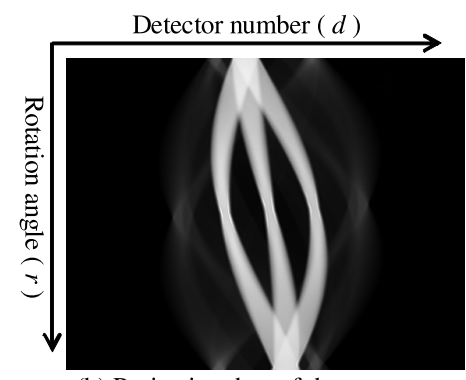

(b) Projection data of the connector.

Fig. 13 Connector for CT imaging and its projection data.

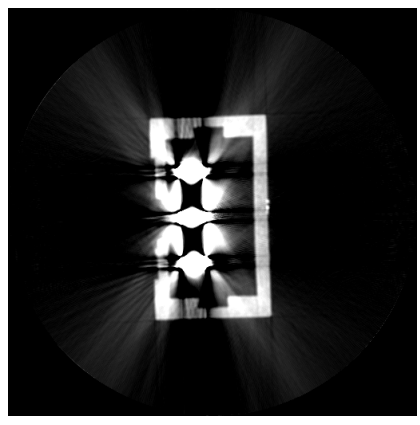

(a) Reconstructed image using the original projection data.

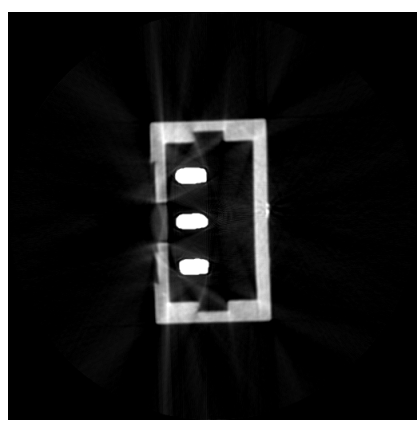

(b) Reconstructed image using the revised projection data.

Fig. 14 Reconstructed images of the connector.

shown in Fig.14(a). A few artifacts remained in the revised projection. Nevertheless, the states of the resin can be adequately observed, as shown in Fig.14(b). This reveals that the proposed method is quite effective for inspecting the fatigue condition of the connector, which is repeatedly inserted and removed.

\section{Discussion}

As mentioned above, the proposed method substantially reduces the metal artifacts and computes precise CT images. However, we may have deformed the shapes of the metallic parts when the scanning object has a more complex structure than the samples above mentioned. For example, the method was applied to a sample with nine steel pegs in an acrylic column as shown in Fig.15(a). The metal artifacts in the image using the original projection data (Fig.15(b)) were almost reduced by the method as shown in Fig.15(c). However, the shapes of the pegs were deformed to polygonal shapes in the image despite their inherent cylindrical shapes. Hence, the proposed method must be further improved.

The phenomenon is because the method uses simple thresholding and converts the projection data to zero in the region where the $\mathrm{x}$-ray transmits the metallic objects. The modified projection data, which is used to compute the shapes of metallic objects, still has inconsistencies and thus, induces an improper reconstruction. Although we used the value of zero in this study, it is possible to calculate consistent values by considering all the projection data for multiple angles. Further research on this point should improve the method so that metal artifacts are reduced and so that the shapes of metallic objects are accurately reconstructed.

\section{Conclusion}

In this paper, we propose a CT image reconstruction algorithm to reduce metal artifacts so that the CT imaging technique can be more widely applied to the non-destructive inspection of devices. The algorithm is based on a direct signal conversion technique of the projection data, which is generally processed only in the CT scanner. We applied the algorithm to two specimens, and revealed the efficiency of the algorithm to reduce the artifacts. Our study indicates that this method is useful for the non-destructive inspection of devices that consist 


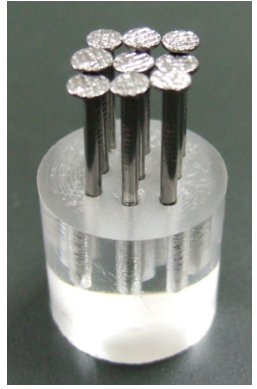

(a) New sample.

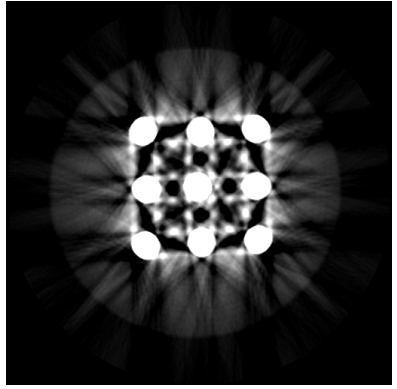

(b) Reconstructed image using the original projection data.

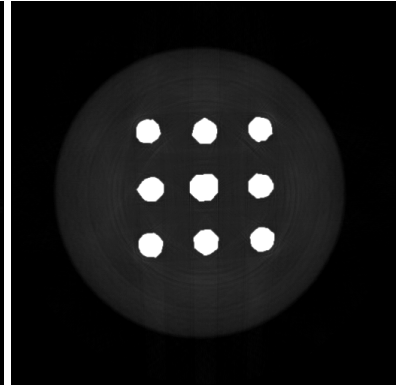

(c) Reconstructed image using the revised projection data.

Fig. 15 Reconstructed images of a new sample.

of resins and metals, like electrical connectors.

However, as mentioned in the previous chapter, this method has a poorer performance according to the scanning object; that is, it is difficult to accurately compute the shapes of the metallic parts when an object has a complex structure. Hence, we are further investigating the extraction technique of the metal region in order to improve the practical utility of this method.

\section{Acknowledgment}

This study was supported by the Industrial Technology Research Grant Program in 2005 from New Energy and Industrial Technology Development Organization (NEDO) of Japan.

\section{References}

( 1 ) Inou, N. et al., Individual Modeling Method Based on the X-ray CT Images (Influence of Partial Volume Effect on the Modeling), Transactions of the Japan Society of Mechanical Engineers, Series A, Vol.69, No.677, (2003), pp.109-114.

( 2 ) Koseki, M. et al., Individual Modeling Method Based on the X-ray CT Images (The Correction Method of CT Values Influenced by Partial Volume Effect), Transactions of the Japan Society of Mechanical Engineers, Series A, Vol.70, No.697, (2004), pp.11701177.

( 3 ) Hashimoto, S. et al., Individual Finite Element Model Based on the X-ray CT Data (Image reconstruction algorithm to reduce metal artifact), Proceedings of the 17th Bioengineering Conference, No.04-48, (2005-1), pp.133-134.

( 4 ) Koseki, M. et al., Development of the Improved X-ray CT Device for Soft Tissues (Influence of $\mathrm{CT}$ artifacts caused by hard tissues), Proceedings of the 18th Bioengineering Conference, No.05-66, (2006-1), pp.307-308.

( 5 ) Hounsfield, G. N., Computed Medical Imaging, Nobel Lectures, Physiology or Medicine 1971-1980, (1992), pp.568-586, World Scientific Publishing Co.

( 6 ) Wang, G. et al., Iterative Deblurring for CT Metal Artifact Reduction, IEEE Transactions on Medical Imaging, Vol.15, No.5, (1996), pp.657-664.

( 7 ) Yokoi, T., Image Reconstruction using Ordered Subsets-Expectation Maximization (OSEM) Algorithm, Japanese Journal of Radiological Technology, Vol.57, No.5, (2001), pp.523-529.

( 8 ) Wei, J. et al., X-ray CT high-density artefact suppression in cryosurgery, Physics in Medicine and Biology, Vol.47, (2002), pp.N319-N326.

( 9 ) Tuy, H. K., A Post-processing Algorithm to Reduce Metallic Clip Artifacts in CT images, European Radiology, Vol.3, (1993), pp.129-134.

(10) Arai, Y. and Suzuki, M., Japanese patent, Publication number: 2001-190550.

(11) Otsu, N., A threshold selection method from gray-level histograms, IEEE Transactions on System, Man and Cybernetics, Vol.9, No.1, (1979), pp.62-66. 